\title{
Congenital varicella syndrome: the evidence for secondary prevention with varicella-zoster immune globulin
}

\author{
Arlan Cohen MD JD, Panagis Moschopoulos PhD, Richard E. Stiehm MD, Gideon Koren MD
}

Competing interests:

Please see end of article for

competing interests.

This article has been peer reviewed.

Correspondence to:

Dr. Gideon Koren,

gkoren@ sickkids.ca

CMAJ 2011. DOI:10.1503 /cmaj.100615

\section{The case}

A 33-year-old woman who was engaged to be married presented for her initial visit with an obstetrician. Her varicella-zoster IgG antibody titer, as measured by latex agglutination, was $<0.91$, which signified nonimmunity. The patient did not know if she had previously had varicella. She had not been vaccinated. Three months later, she became pregnant. She was told to avoid crowds of children and to seek vaccination after her delivery.

At 13 weeks of gestation, the patient was visited by her mother, who complained of severe pain over one side of her scalp. When the patient moved her mother's hair to see what was causing the pain, she felt her mother's hair to be wet to her touch and saw multiple small blisters. An immediate visit to an internal medicine specialist confirmed a diagnosis of herpes zoster infection in the patient's mother. The patient was sent within 18 hours of her mother's arrival and her potential exposure to zoster to her obstetrician. Varicella-zoster immune globulin (VZIG) was not given.

Three weeks later, the patient broke out in a varicella rash and was treated with acyclovir.

\section{KEY POINTS}

- In $1 \%-2 \%$ of cases, maternal chickenpox may result in congenital varicella syndrome, which is associated with severe, lifelong disabilities.

- Evidence from prospective cohort studies suggests high rates of protection against fetal infection and congenital varicella syndrome in mothers with chicken pox who received varicella-zoster immune globulin (VZIG).

- Given advice by several major guidelines that VZIG can improve maternal morbidity but not fetal outcome, clinicians may not appreciate the urgency of giving VZIG to nonimmune pregnant patients on reported contact with varicella.

- Guidelines should be revised to reflect the available evidence on the effectiveness of VZIG in preventing congenital varicella syndrome.

She recovered completely. The patient then began follow-up with a maternal-fetal medicine specialist, seeking information about whether her fetus was infected with varicella and whether the fetus had congenital varicella syndrome.

Repeated sonographic examinations at 19 and 24 weeks of gestation were interpreted as showing bilateral clubfeet, with no other abnormality. Amniocentesis was not performed. At 32 weeks of gestation, ultrasonography showed intrauterine growth retardation, polyhydramnios and stippling of the fetal liver. An amniocentesis performed at that time was culture-negative for varicella virus, but results of polymerase chain reaction testing showed varicella-zoster DNA. No cytomegalovirus DNA was found in the amniotic fluid. Fetal cells showed a normal karyotype. No cause for the findings on ultrasound, other than likely congenital varicella syndrome, was found. Because of worsening intrauterine growth retardation, the baby was delivered by cesarean section at 36 weeks of gestation.

The baby was born with bilateral clubfeet, partial aplasia of the right lower extremity, microgastria, severe reflux, an absent gag reflex and profound oral aversion (i.e., refusal to feed). Over the subsequent three years, she has had repeated episodes of aspiration pneumonia and has required feeding through gastrostomy and jejunostomy tubes, a permanent tracheostomy and 24-hour oxygen therapy. She has had multiple episodes of cutaneous zoster infection and of viral and bacterial pneumonia, requiring prolonged periods on a ventilator. She has had a gastric fundoplication and surgical repair of her clubfeet. Within the last six months before time of writing, she has been found to have partial defects of both cellular and humoural immunity, with antibody responses to protein antigens but not to 
polysaccharide antigens, and with selective defects in cellular immunity, including subnormal cellular response to varicella-zoster virus. She continues to be unable to handle oral food or oral secretions and has experienced apneic spells, some involving loss of consciousness. She has also had episodes of cyclic vomiting, lasting hours to days, which have not been responsive to medications. The child has learned to communicate by using sign language, and she has a vocabulary of 20 words.

The patient and family launched a malpractice lawsuit, which was settled in their favour.

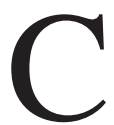

ongenital varicella syndrome is the result of serious infection and strikes $1 \%-3 \%$ of fetuses whose susceptible mothers have contracted chicken pox in pregnancy. ${ }^{1,2}$ The greatest risk of developing congenital varicella syndrome is apparent when a nonimmune pregnant woman is infected during the thirteenth to twentieth week of pregnancy. ${ }^{3}$ Primary prevention of congenital varicella syndrome consists of effective vaccination of the mother before conception, while secondary prevention involves the use in susceptible pregnant women of varicella-zoster immune globulin, a specific $\operatorname{IgG}$ antibody against varicella-zoster virus, after exposure.

We systematically review the existing evidence for secondary prevention of chronic varicella syndrome by varicella-zoster immune globulin after maternal exposure to varicellazoster virus during pregnancy.

\section{Methods}

To identify all articles examining the evidence related to whether varicella-zoster immune globulin prevents congenital varicella syndrome, we completed a computerized search of Medline, EMBASE, the Cumulative Index to Nursing and Allied Health Literature (CINAHL) and the Cochrane library from inception to July 1, 2010. We supplemented the search by investigating relevant references from published reviews. There were no exclusionary criteria by language of publication. Search terms included "pregnancy and chickenpox" or "varicella zoster," and "congenital varicella syndrome" or "VZIG." We included all original research involving humans and related to prevention of congenital varicella syndrome by varicella-zoster immune globulin. We excluded animal studies, experimental laboratory studies, case reports and editorials.

We subsequently reviewed the most recent practice guidelines on chickenpox in pregnancy by the American College of Obstetrics and
Gynecology, the Society of Obstetrics and Gynaecology of Canada, the US Food and Drug Administration, the Centers for Disease Control and Prevention and the American Academy of Pediatrics. We aimed to verify whether the existing evidence supporting secondary prevention of congenital varicella syndrome by varicellazoster immune globulin has been incorporated into the guidelines.

The included papers were examined for strength of evidence based on the grading recommendations of Woolf and colleagues. ${ }^{4}$ Our search identified 232 articles, of which three ultimately met our inclusion criteria and contained original data on prevention of congenital varicella syndrome by varicella-zoster immune globulin. $3 ., 5,6$

\section{What evidence supports prevention of congenital varicella syndrome by varicella-zoster immune globulin?}

Three events must occur before a fetus contracts congenital varicella syndrome. They are clinical infection in the mother, viral infection in the fetus and development of congenital varicella syndrome in the infected fetus. We present the existing evidence synthesized into an overall estimate of the protective effect of varicellazoster immune globulin.

\section{Protection of the mother from development of clinical varicella}

A number of studies, which date back half a century, have shown that the administration of varicella-zoster immune globulin is effective in decreasing rates of contraction of clinically evident chicken pox in exposed immunocompetent patients by $40 \%-50 \%$ or more and in attenuating the severity of varicella infection in those who contract it, whether they are immunocompetent or immunocompromised. ${ }^{7-13}$ Varicella-zoster immune globulin neutralizes free virus and is effective even at very low levels in antibodydependent cellular cytotoxicity. ${ }^{8}$ McGregor and colleagues, ${ }^{9}$ for example, found that $80 \%$ of seronegative pregnant women with exposure who received varicella-zoster immune globulin remained symptom-free, whereas $89 \%$ of untreated control patients developed clinical illness. However, the sample size of the study and the size of each study arm were not reported. Brunell and colleagues, ${ }^{7}$ in a study done over 40 years ago, found that all of six immunocompetent children exposed to varicella virus who did not receive varicella-zoster immune globulin contracted varicella, whereas none of the six 
treated with varicella-zoster immune globulin developed the disease or antibodies against the disease. Using the grading scheme of the Canadian Task Force on Preventive Health Care, ${ }^{4}$ we graded the existing evidence supporting the use of varicella-zoster immune globulin in protecting the mother from varicella as Level I (i.e., one or more properly randomized controlled trial).

There is only one reported case of a child developing typical congenital varicella syndrome when the previously nonimmune mother did not show clinically evident varicella. ${ }^{10}$ Maternal viremia mediates the production of clinical symptoms. With absent clinical symptoms, maternal viremia is likely to be absent or greatly diminished, even if production of new antivaricella antibodies shows maternal infection. Protection of the previously nonimmune mother from clinical disease, with very rare exceptions, appears to protect the fetus from congenital varicella syndrome.

\section{Protection of the fetus from infection}

Not all fetuses of mothers who develop clinical varicella during pregnancy become infected with the virus. The infection rate of fetuses has been reported to range from $12 \%-30 \% .^{3}$ varicellazoster immune globulin attenuates clinical disease in the mother not necessarily by eradicating viremia but often by lessening it. Given that the large majority of fetuses apparently are not infected even when the mother has a "full" viral load, it is biologically plausible that varicellazoster immune globulin, which may lessen the viral load to which the fetus is exposed, would lower the fetal infection rate. Only one study reported on the rates of specific varicella IgM antibodies in the cord blood of babies whose mothers contracted varicella during pregnancy. The study's results showed decrease from $12.4 \%$ $(76 / 615)$ in cases where the mother was not treated with varicella-zoster immune globulin to $1.1 \%(1 / 89)$ in babies whose mothers received timely varicella-zoster immune globulin after exposure $(p<0.01){ }^{3}$

Using the grading scheme of the Canadian Task Force on Preventive Health Care, ${ }^{4}$ we graded the existing evidence for protection from infection of the fetus by varicella-zoster immune globulin as Level II-2 (i.e., evidence from cohort or case-control analytic studies).

\section{Prevention of congenital varicella syndrome}

It has been argued that decrease in the rate of fetal infection is only an indirect indication that varicella-zoster immune globulin may prevent congenital varicella syndrome and does not prove that this protection is real.
Direct evidence for the effect of varicellazoster immune globulin on the incidence of congenital varicella syndrome comes from the following three published studies: the large study based in Germany and the United Kingdom and cited above, ${ }^{3}$ the extension of that study based on analysis of data collected during five additional years ${ }^{5}$ and one other prospective study by Mouly and colleagues. ${ }^{6}$ These together comprise the only prospective studies of the incidence of congenital varicella syndrome that isolated the administration of varicella-zoster immune globulin to the mother as a variable. All three studies contained prospective cohort data. No randomized controlled trials have been reported. Using the grading scheme of the Canadian Task Force on Preventive Health Care, ${ }^{4}$ we graded the quality of evidence in these three studies as Level II-2 (i.e., evidence from cohort or case-control analytic studies).

In the first study, Enders and colleagues reported that the incidence of congenital varicella syndrome among babies treated with varicella-zoster immune globulin whose mothers contracted varicella during the thirteenth to twentieth week of pregnancy was $0 / 97$. In contrast, the incidence of congenital varicella syndrome among babies of women who contracted varicella during the same time period, but who did not receive varicella-zoster immune globulin, was 7/351 $(1.99 \%)(p<0.01) .^{3}$ Enders subsequently extended this study with data derived from her Stuttgart site, collected from 1994 to 1999. ${ }^{.1}$ An additional 11 pregnant women with varicella during pregnancy who had received varicella-zoster immune globulin were followed; none of the babies developed congenital varicella syndrome. By contrast, another 82 patients were followed who contracted varicella during weeks 13-20 but were not given varicella-zoster immune globulin; four instances of congenital varicella syndrome occurred.

Finally, Mouly and colleagues reported on 107 women who contracted varicella in the first 24 weeks of pregnancy. ${ }^{12}$ Of these, 99 were followed through to term. Thirty-four of the women in the study were given varicella-zoster immune globulin. None of their 34 babies had congenital varicella syndrome. Of the remaining presumably 65 women who did not receive varicellazoster immune globulin, three of their babies developed congenital varicella syndrome.

Combining the data from these three studies, $3,5,6$ a total of 640 pregnant women diagnosed with varicella were followed through delivery. Of those, 142 were treated with varicella-zoster immune globulin, with congenital varicella syndrome not found in any fetus or infant born to 
these mothers. By contrast, of the remaining 498 who were not treated with varicella-zoster immune globulin, 14 infants were diagnosed with congenital varicella syndrome $(2.81 \%)$ ( $p<0.01$ by Fisher exact test).

The known risks associated with maternal receipt of varicella-zoster immune globulin are remote and mostly related to allergic reactions, anaphylaxis or local irritation. ${ }^{14}$ The cost of one vial of varicella-zoster immune globulin is around \$400 (Cdn). ${ }^{15}$ Formal cost-benefit analysis of varicella-zoster immune globulin in pregnancy has been calculated only for prevention of maternal morbidity and mortality, but not for prevention of congenital varicella syndrome. ${ }^{16}$

\section{What do practice guidelines recommend?}

In the United States, the American College of Obstetrics and Gynecology Practice Bulletin \#20, entitled "Prenatal viral and parasitic infections" and published in September 2000, ${ }^{17}$ states "If exposure does occur, prophylactic intervention with varicella-zoster immune globulin early in the incubation period can prevent or attenuate the disease manifestations of VZV in susceptible contacts at high risk from this infection."

In the same paragraph, however, this bulletin states that "maternal administration of varicellazoster immune globulin does not ameliorate or prevent fetal infection." It goes further to state explicitly that "there is no protective effect." ${ }^{\text {" }}$ In this bulletin, which contains 131 citations of the literature, there is no citation to support the assertion of lack of protective effect of varicella-zoster immune globulin. Existing evidence that prevention of clinical disease in the mother may have a protective effect on the fetus is not considered. The bulletin cites the initial Enders study ${ }^{3}$ only for the overall incidence of congenital varicella syndrome, not mentioning the protective fetal effect nor citing the later published data. ${ }^{5.6}$

The guidelines of the Centers for Disease Control and Prevention ${ }^{18}$ include the following statement: "Because pregnant women might be at higher risk for severe varicella and complications, use of varicella-zoster immune globulin (VZIG) should be strongly considered for pregnant women without evidence of immunity who have been exposed. However, administration of VZIG to pregnant women has not been found to prevent viremia, and therefore does not prevent fetal infection, congenital varicella, or neonatal varicella. The primary indication for VZIG in pregnant women is to prevent complications in the mother rather than to protect the fetus." Similarly, the
2008 Federal Drug Administration guidelines assert that "there is no evidence that administration of VZIG to a susceptible pregnant woman will prevent viremia, fetal infection, or congenital varicella syndrome. Therefore the primary indication for VZIG in pregnant women is to prevent complications of varicella in a susceptible adult patient rather than to prevent intrauterine infection." ${ }^{\prime 19}$

These statements appear to contradict previous Centers for Disease Control and Prevention publications acknowledging, for at least twentyfive years, that varicella-zoster immune globulin prevents clinical symptoms in a substantial percentage of exposed patients and mitigates the disease in those in whom clinical symptoms were not entirely prevented. ${ }^{20}$

Last, the American Academy of Pediatrics has adopted the Centers for Disease Control and Prevention perspective, with similar recommendations. ${ }^{21}$

In Canada, the 2009 Practice Guidelines on Immunization in Pregnancy by the Society of Obstetricians and Gynecologists of Canada mention fetal protection by varicella-zoster immune globulin as a theoretical benefit, but do not discuss the available evidence for prevention of fetal infection. ${ }^{22}$ The guidelines state that "susceptible women should then be offered varicellazoster immune globulin within 96 hours of exposure in an attempt to prevent the disease or reduce the severity of the infection in the mother ... Although there may also be some benefit to the fetus, this remains to be investigated in a clinical trial." ${ }^{21}$

\section{What should clinicians do?}

Prospective, randomized, double-blind, controlled studies are the gold standard in clinical science. For diseases as rare as congenital varicella syndrome, the likelihood that any such study will be sufficiently large to have the power to make absolute conclusions about the effect of varicella-zoster immune globulin is prohibitively small. Further, the available data to date on the beneficial effect of varicella-zoster immune globulin for the exposed nonimmune pregnant mother and her baby would render such a study ethically unacceptable. Given this reality, it is important for clinicians to be familiar with the available evidence, even if, on the scale of strength, it does not compare with randomized controlled trials. Although from regulatory perspectives, without appropriate clinical trials, none of the varicella immunoglobulin products can be labelled as "preventing congenital varicella syndrome," the existing evidence should not be ignored by clinicians. 
It is likely that guidelines have not made stronger statements about the effectiveness of varicella-zoster immune globulin in secondary prevention of congenital varicella syndrome because the existing evidence did not come from a randomized controlled trial. It is "off label" use, and available evidence of efficacy, effectiveness and safety should be addressed by professional societies and public health authorities.

Although the existing data on prevention of fetal infection and congenital varicella syndrome are not perfect, randomized controlled trials are unlikely to be conducted for this relatively rare medical event. The analysis presented here indicates a very high likelihood that varicella-zoster immune globulin substantially protects the fetus from congenital varicella syndrome at each of the steps in the development of final organ damage if the nonimmune mother is treated within 96 hours of exposure. Of importance, the adverse effects associated with varicella-zoster immune globulin (e.g., allergic reactions, anaphylaxis) are minimal. ${ }^{14}$

Unless guidelines are revised to reflect the inherent uncertainty in the evidence base and the fact that definitive studies are unlikely to be conducted, health care providers (and their nonimmune pregnant patients) may not appreciate the urgency of administering varicella-zoster immune globulin immediately upon reported contact with varicella. These patients and their babies may be put at avoidable risk because of assumptions regarding the ineffectiveness of varicella-zoster immune globulin in affording fetal protection.

\section{References}

1. Alkalay AL, Pomerance JJ, Rimoin DI. Fetal varicella syndrome. J Pediatr 1987;111:320-3.

2. Tan MP, Koren G. Chickenpox in pregnancy: revisited. Reprod Toxicol 2006;21:410-20.

3. Enders G, Miller E, Cradock-Watson J, et al. Consequences of varicella and herpes zoster in pregnancy: prospective study of 1739 cases. Lancet 1994;343:1548-51.

4. Canadian Task Force on Preventive Health Care. New grades for recommendations from the Canadian Task Force on Preventive Health Care. CMAJ 2003;169:207-8.

5. Enders G, Miller E. Varicella and herpes zoster in pregnancy and the newborn. In: Varicella- zoster virus: virology and clinical management. Cambridge University Press 2000; 317- 47.

6. Mouly F, Mirlesse V, Meritet J, et al. Prenatal diagnosis of fetal varicella-zoster virus infection with polymerase chain reaction of amniotic fluid in 107 cases. Am J Obstet Gynecol 1997;177:894-8.

7. Brunell PA, Ross A, Miller H, et al. Prevention of varicella by zoster immune globulin. N Engl J Med 1969;280:1191-4.

8. Taylor-Robinson D. Chickenpox and herpez zoster. III. Tissue culture studies. Br J Exp Pathol 1959;40:521-32.

9. McGregor JA, Madinger AJ. Varicella zoster in pregnancy. In: Infection protocols for obstetrics and gynecology. Montvale (NJ): Medical Economics Publishing; 1992. p. 94-100.

10. Al-Katawee YA, Al-Hasoun YA, Taha MN, et al. Congenital varicella-zoster virus infection. A rare case of severe brain and ocular malformations without limb or cutaneous involvement in a newborn after maternal subclinical infection. Saudi Med J 2005;26:869-71.

11. Orenstein WA, Heyman DL, Ellis RJ, et al. Prophylaxis of varicella in high risk children: response effect of zoster immune globulin. J Pediatr 1981;98:368-73.

12. Brunell PA, Gershon AA, Hughes WT, et al. Prevention of varicella in high-risk children; a collaborative study. Pediatrics 1972;50:718-22.

13. Gershon AA, Steinberg S, Brunell PA. Zoster immune globulin: a further assessment. N Engl J Med 1974;290:243-5.

14. Paryani SG, Arvin AM, Koropchak CM, et al. Comparison of varicella zoster antibody titers in patients given intravenous immune serum globulin or varicella zoster immune globulin. Pediatr 1984;105:200-5.

15. Thoughton J, Crealey G, Crawford V, et al. VZIG administration in pregnant women exposed to chickenpox. Proceedings of the 18th European Congress of Clinical Microbiology and Infectious Diseases; 2008 Apr. 19-22; Barcelona, Spain. Abstract no. 0254. Munich: European Society of Clinical Microbiology and Infectious Diseases; 2008.

16. Rouse DJ, Gardner M, Allen SJ, et al. Management of the presumed susceptible varicella exposed gravida: a cost effectiveness/cost-benefit analysis. Obstet Gynecol 1996;87:932-6.

17. ACOG practice bulletin. Perinatal viral and parasitic infections. Number 20, Sept. 2000. (Replaces educational bulletin number 177, February 1993). American College of Obstetrics and Gynecologists. Int J Gynaecol Obstet. 2002;76:95-107

18. Prevention of varicella: recommendations of the Advisory Committee on Immunization Practices (ACIP). Centers for Disease Control and Prevention. MMWR Recomm Rep 1996;45:1-36.

19. Vaccines, blood and biologics: varicella zoster immune globulin (VZIG), anticipated short supply and alternate product availability under an investigational new drug application expanded acccess protocol. Silver Spring (MD): U.S. Food and Drug Administration; 2008. Available: www.fda.gov/BiologicsBlood Vaccines/SafetyAvailability/ucm 176029.htm (accessed 2010 Dec. 22).

20. CDC. Varicella Zoster Immune Globulin for the prevention of chickenpox. MMWR Morb Mortal Wkly Rep 1984;33:84-90.

21. Pickering LK, Baker CJ, Kimberling DW, et al., editors. 2009 Red Book Report of the Committee on Infectious Diseases. Elk Grove Village (IL): American Academy of Pediatrics; 2009.

22. Gruslin A. Steben M, Halperin S., et al. SOGC Clinical Practice Guidelines. Immunization in pregnancy. JOGC 2009;31:10851092. Available: www.sogc.org/guidelines/documents/gui236 CPG0911.pdf (accessed 2010 Dec. 22).

Competing interests: Arlan Cohen was the attorney for a malpractice suit launched by the patient and family described in this paper. The lawsuit was settled in favour of his client before this paper was written. He was not involved in the patient's clinical care. Richard Stiehm has provided expert testimony for malpractice cases on behalf of UCLA. He has served on speaker bureaus of CSL Behring and Baxter Laboratories and received monetary compensation for travel expenses from both firms for conferences on intravenous immune globulin (IVIG). No competing interests declared by Panagis Moschopoulos and Gideon Koren.

Affiliations: From the law offices of Arlan A. Cohen, the Department of Pediatrics (Stiehm), University of California, Los Angeles, USA; the Department of Mathematical Sciences (Moschopoulos), University of Texas, El Paso, USA; and the Motherisk Program, Department of Pediatrics (Koren), University of Toronto, Toronto, Ont.

Contributors: Arlan Cohen and Gideon Koren contributed to the article design, review of the literature, and drafting and revision of the manuscript. Richard Stiehm provided critical analysis of varicella zoster effects in pregnancy and reviewed the manuscript. Panagis Moschopoulos provided statistical advice and analyses. All of the authors approved the final version of the manuscript submitted for publication. 